Article

\title{
Scandium Decoration of Boron Doped Porous Graphene for High-Capacity Hydrogen Storage
}

\author{
Jing Wang ${ }^{1,2}$, Yuhong Chen ${ }^{1,2, *}$, Lihua Yuan ${ }^{2}$, Meiling Zhang ${ }^{2}$ and Cairong Zhang ${ }^{1,2}$ (I) \\ 1 State Key Laboratory of Advanced Processing and Recycling of Non-Ferrous Metals, Lanzhou University of \\ Technology, Lanzhou 730050, China \\ 2 School of Science, Lanzhou University of Technology, Lanzhou 730050, China \\ * Correspondence: chenyh@lut.cn; Tel.: +86-931-297-3780
}

Academic Editors: Takayuki Ichikawa and Ankur Jain

Received: 10 May 2019; Accepted: 25 June 2019; Published: 27 June 2019

check for updates

\begin{abstract}
The hydrogen storage properties of the Scandium (Sc) atom modified Boron (B) doped porous graphene (PG) system were studied based on the density functional theory (DFT). For a single Sc atom, the most stable adsorption position on B-PG is the boron-carbon hexagon center after doping with the $\mathrm{B}$ atom. The corresponding adsorption energy of $\mathrm{Sc}$ atoms was $-4.004 \mathrm{eV}$. Meanwhile, five $\mathrm{H}_{2}$ molecules could be adsorbed around a Sc atom with the average adsorption energy of $-0.515 \mathrm{eV} / \mathrm{H}_{2}$. Analyzing the density of states (DOS) and the charge population of the system, the adsorption of $\mathrm{H}_{2}$ molecules in Sc-B/PG system is mainly attributed to an orbital interaction between $\mathrm{H}$ and $\mathrm{Sc}$ atoms. For the $\mathrm{H}_{2}$ adsorption, the Coulomb attraction between $\mathrm{H}_{2}$ molecules (negatively charged) and $\mathrm{Sc}$ atoms (positively charged) also played a critical role. The largest hydrogen storage capacity structure was two Sc atoms located at two sides of the boron-carbon hexagon center in the Sc-B/PG system. Notably, the theoretical hydrogen storage capacity was $9.13 \mathrm{wt} . \%$ with an average adsorption energy of $-0.225 \mathrm{eV} / \mathrm{H}_{2}$. B doped PG prevents the Sc atom aggregating and improves the hydrogen storage effectively because it can increase the adsorption energy of the $\mathrm{Sc}$ atom and $\mathrm{H}_{2}$ molecule.
\end{abstract}

Keywords: first principles; porous graphene; B doped Sc modified PG; hydrogen storage

\section{Introduction}

The development of human society faces the severe challenge of environmental pollution, which is urgently needed to explore more ideal energy materials to meet the growing energy demand [1-3]. Hydrogen $\left(\mathrm{H}_{2}\right)$ is used as a promising energy carrier because of recycling, no pollution, high energy density and high calorific value [4-6]. Graphene is a two-dimensional material with a hexagonal honeycomb structure composed of sp2 hybrid orbits. Graphene-based materials possess large specific surface area, good adsorption kinetics, low density, high chemical stability and reversible hydrogen storage, which is a potential candidate in many areas, such as solid-state hydrogen storage, electronics and sensors [7-14]. However, due to a large surface inertness and weak binding ability to $\mathrm{H}_{2}$ molecules, clean graphene is difficult to become a promising hydrogen storage material [15-19].

Recently, transition metal atoms $[20,21]$ modified graphene has attracted researchers' attention since significantly increasing the adsorption energy of $\mathrm{H}_{2}$ molecules. Faye et al. [22] found that $\mathrm{Pd}$ double-sided modified graphene could adsorb up to eight $\mathrm{H}_{2}$ molecules using local density approximation (LDA) of Dmol3 software. The transition metal atoms Sc, Ti and V decorated on both sides of graphene could adsorb four $\mathrm{H}_{2}$ molecules with an average adsorption energy of $0.300-0.500 \mathrm{eV} / \mathrm{H}_{2}$ [23].

Yet the transition metal atom modified graphene is prone to aggregation, which reduces the adsorption site of $\mathrm{H}_{2}$ and weakens the hydrogen storage performance of graphene. Investigations have shown that the introduction of impurity atoms or vacancies can prevent the agglomeration of 
transition metal atoms and increase the hydrogen storage capacity of graphene [24]. There are two effective means available at present. The first method is a non-metal atom such as B, N etc. doped graphene [25-28]. Since the B atom has an empty p orbit, it can be used as an electron acceptor to obtain the electrons of other modified atoms, enhancing the adsorption energy of the modified atom [29]. Meanwhile, B-doped graphene has been successfully synthesized in experiments [30]. To introduce pore defects into graphene is another method [31,32]. Compared with non-defective graphene, there is no agglomeration between the metals because the metal atoms are closer to the substrate with a high metal binding energy. Moreover, the porous material adsorbs $\mathrm{H}_{2}$ through Van der Waals (VDW) interactions between the system and the $\mathrm{H}_{2}$ molecule, which is physical adsorption. Porous graphene (PG) [33] is a two-dimensional material synthesized in experiments with high specific surface area, which can store hydrogen efficiently [34]. Du [2] used local density approximation (LDA) of the VASP code to study the adsorption of $\mathrm{H}_{2}$ on the $\mathrm{Li}$ atom modified porous graphene. The hydrogen storage capacity of the system can reach $12.00 \mathrm{wt} . \%$, and the average adsorption energy is about $0.243 \mathrm{eV}$. However, the LDA functional will overestimate the average binding energy of $\mathrm{H}_{2}$ molecules. Additionally, Reunchan [35] and Yuan [36] studied the hydrogen storage properties of Ca and Y atom-modified porous graphene by generalized gradient approximation (GGA) functional theory.

The research found that the combination of these two methods could avoid the aggregation of transition metal atoms on the PG surface, meet the requirement of the U.S. Department of Energy (DOE) for hydrogen storage, but also make the adsorption energy of $\mathrm{H}_{2}$ molecules in the range of reversible hydrogen storage [37,38]. Simultaneously, Sc modified porous graphene possessed a larger amount of hydrogen storage because of the lightest atomic mass in the transition metal atom. However, there are only a few reports on the adsorption mechanism and hydrogen storage capacity of Sc decorated B-doped PG.

Herein, we combined the Sc modification and the B doping methods to modify the porous graphene. In this paper, the most stable adsorption structure and adsorption energy of Sc decorated B-doped PG were manipulated via a first-principles calculation. In addition to this, the performance and mechanism of the $\mathrm{H}_{2}$ molecule adsorption in Sc-modified B-PG system were analyzed deeply. Based on the results, the largest hydrogen storage capacity structure was two Sc atoms located at two sides of the boron-carbon hexagon center in the Sc-B/PG system. Theoretically, the hydrogen storage capacity was $9.13 \mathrm{wt}$ \% with an average adsorption energy of $-0.225 \mathrm{eV} / \mathrm{H}_{2}$. B doped PG prevents the Sc atom aggregating and improves the hydrogen storage effectively because it can increase the adsorption energy of the $\mathrm{Sc}$ atom and $\mathrm{H}_{2}$ molecule.

\section{Calculation Details}

Based on the density functional theory, the GGA function of the CASTEP software package [39] was used to select the PBE exchange correlation gradient correction [40]. The Van der Waals correction (DFT-D method) was used in order to avoid the GGA function underestimation of energy in the calculation. All the atoms in the calculation were completely relaxed. The convergence criteria of the optimized structure were as follows: Each atom was subjected to a force below $0.01 \mathrm{eV} / \AA$; the energy convergence tolerance was lower than $5.0 \times 10^{-6} \mathrm{eV} /$ atom; the self-consistent field convergence threshold was selected as $1.0 \times 10^{-6} \mathrm{eV} /$ atom between two steps. The vacuum layer was selected as $20 \AA$ along the $\mathrm{Z}$ direction of the PG to eliminate the interlayer interaction. Considering the calculation accuracy and calculation cost, the cutoff energy was selected as $500 \mathrm{eV}$, and the K-point in the Brillouin zone was $7 \times 7 \times 1$.

Formula (1) is used to define the average binding energy $\left(\bar{E}_{b}\right)$ of Sc atoms on B-PG:

$$
\bar{E}_{b}=\left[E_{S c+B / P G}-E_{B / P G}-n E_{S c}\right] / n
$$


among which $E_{S c+B / P G}, E_{B / P G}$ and $E_{S c}$ represent the energy in the Sc-modified B-doped PG system, the B-doped PG system and a free Sc atom, respectively. Additionally, $\mathrm{n}$ represents the number of adsorbed Sc atoms.

The adsorption energy $\left(E_{a d}\right)$ and average adsorption energy $\left(\bar{E}_{a d}\right)$ of the $\mathrm{H}_{2}$ molecule is defined as:

$$
\begin{gathered}
E_{a d}=\left[E_{i H_{2}+S c+B / P G}-E_{(i-1) H_{2}+S c+B / P G}-E_{H_{2}}\right] \\
\bar{E}_{a d}=\left[E_{i H_{2}+S c+B / P G}-E_{S c+B / P G}-i E_{H_{2}}\right] / i
\end{gathered}
$$

where $E_{i H_{2}+S c+B / P G}, E_{(i-1) H_{2}+S c+B / P G}$ and $E_{H_{2}}$ respectively represent the total energy of the system with $\mathrm{i}$, (i-1) $\mathrm{H}_{2}$ molecules adsorbed and the total energy of a free $\mathrm{H}_{2}$ molecule.

The formation energy [41] is defined as:

$$
E_{f}=\left[E_{B / P G}-n_{C} \mu_{C}-n_{H} \mu_{H}-n_{B} \mu_{B}\right] /\left[n_{C}+n_{H}+n_{B}\right]
$$

where $E_{B / P G}$ is the energy of the doped porous graphene, $n_{C}, n_{H}$ and $n_{B}$ are the number of $C, H$ and $B$ atoms in the doped porous graphene, respectively. $\mu_{C}, \mu_{H}, \mu_{B}$ denote the chemical potential of $\mathrm{C}, \mathrm{H}, \mathrm{B}$ atoms, respectively $\left(\mu_{\mathrm{C}}=E_{\text {graphene }} /\right.$ number of carbon atoms in graphene, $\mu_{H}=E_{H_{2}} / 2$, $\left.\mu_{B}=E_{B_{12} \text { crystal }} / 12\right)$.

\section{Results and Discussion}

\subsection{Single B Atom Doped Stable Position}

Firstly, six structures of B doped PG were considered. The optimized structures and lattice parameters of 1 6 B-doped PG systems are shown in Table 1. The calculation results show that: As the number of doped B atoms increased, the formation of the doping system became larger and larger, and the energy barrier to be overcome by the doping increased, making doping difficult, which was consistent with Lu et al. [38]. Therefore, we only considered the case of doping one B atom. The optimized structure of a single B-doped PG system is shown in Table 1. The lattice constant was $7.57 \AA$, the B-H bond length was $1.19 \AA$, and the formation energy required for doping was $121.3 \mathrm{meV} /$ atom. The lattice constant of $7.57 \AA$ was significantly higher than that of clean porous graphene $7.49 \AA$ [37], but it was consistent with the lattice constant of $7.57 \AA$ obtained by Lu [38]. The increase in the lattice constant was due to the radius of the $B$ atom being greater than the radius of the replaced $\mathrm{C}$ atom. The measured B-H bond length of $1.19 \AA$ was completely consistent with that of Lu et al. [38]. The formation energy of the B-PG doping system was $121.30 \mathrm{meV} / \mathrm{atom}$, which was also close to the result of $120.20 \mathrm{meV} /$ atom [38], indicating that the calculation method and accuracy were feasible.

In order to study the effect of B atom doping on the performance of porous graphene, the density of states of a single B atom doped porous graphene system was calculated. The results are shown in Figure 1. It can be seen that there was an energy band passing near the Fermi surface after doping $\mathrm{B}$ atoms compared with the clean porous graphene density of states; the change of density of states near the Fermi surface of the doping system mainly came from the contribution of the $2 p$ orbit of the B atom. In addition, the $2 p$ orbit of the $B$ atom overlapped with the porous graphene in the vicinity of the Fermi surface. We obtained the B-PG system band gap of $0.349 \mathrm{eV}$, which was much smaller than the undoped PG's band gap value of $2.399 \mathrm{eV}$. From Figure 1, in the interval of $-3.0 \sim-2.0 \mathrm{eV}$, the peak of PG shifted to the right due to the influence of the B atom $2 p$ orbit. The doping system electrons were close to the Fermi level. This indicates that B atom doping increases the chemical activity of the PG system to some extent and can improve the hydrogen storage performance of the PG system. 
Table 1. Optimized geometries, lattice constants, representative B-H bond lengths, and formation energies of the B-doped porous graphene (PG) system. Note: Gray, white and pink balls represent C, H and $\mathrm{B}$ atoms, respectively.

\begin{tabular}{|c|c|c|c|c|c|c|}
\hline & 1B/PG & 2B/PG & 3B/PG & 4B/PG & $5 B / P G$ & $6 B / P G$ \\
\hline \multicolumn{7}{|l|}{ Top View } \\
\hline a $(\AA)$ & 7.57 & 7.62 & 7.72 & 7.79 & 7.88 & 7.95 \\
\hline$d_{B-H}(\AA)$ & 1.19 & 1.19 & 1.20 & 1.19 & 1.20 & 1.21 \\
\hline$E_{f}(\mathrm{meV} /$ atom $)$ & 121.30 & 168.03 & 247.61 & 291.03 & 377.73 & 440.99 \\
\hline
\end{tabular}

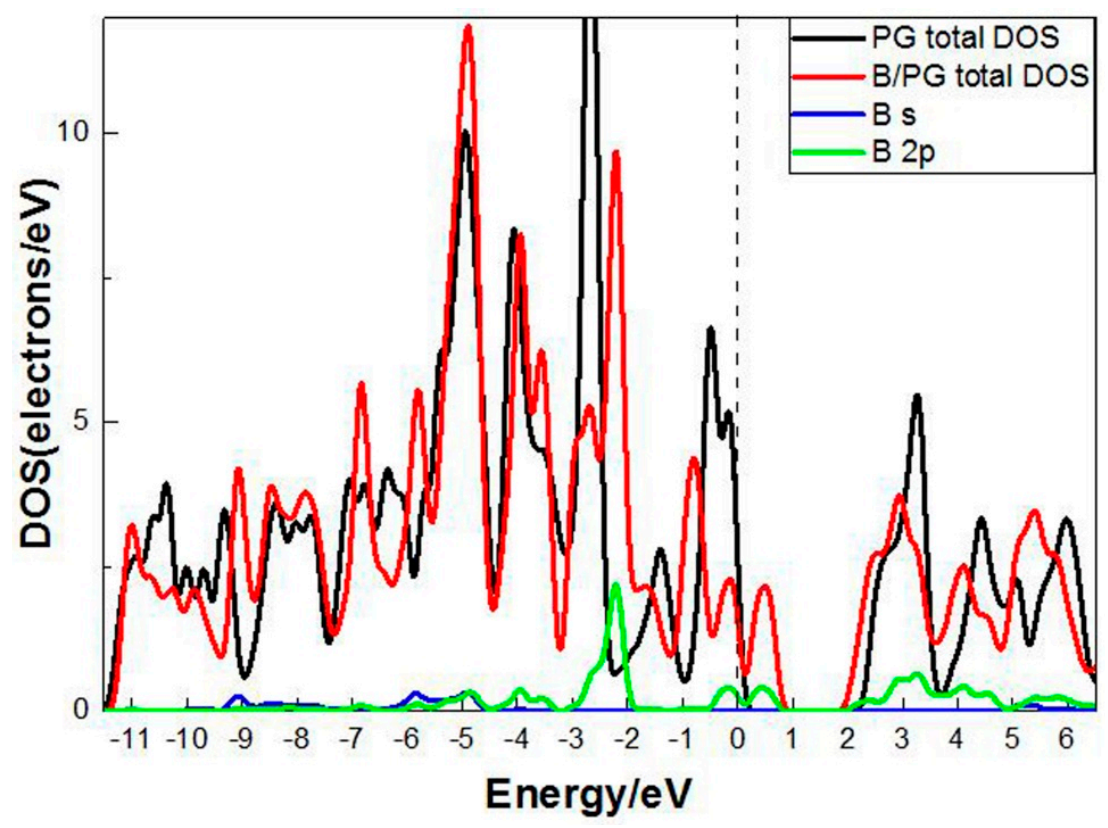

Figure 1. DOS of the porous graphene system with and without B-doped.

\subsection{Single Sc Atom Modified B-PG System}

\subsubsection{Adsorption Structure of Single Sc Atom Modified B-PG}

First, the adsorption of Sc atoms on porous graphene doped with a B atom was investigated. When only one Sc atom was modified, six different adsorption sites were considered. As shown in Table 1 on 1B/PG, adsorption sites were named 1, 2, 3, 4, 5, and 6, which represented the hole position of the $\mathrm{C}$ ring, $\mathrm{C}-\mathrm{C}$ bridge, half $\mathrm{C}$ ring hole position, large hexagon hole position, $\mathrm{C}$ top position and $\mathrm{B}$ top position. Results indicated that position 1 was the most easily adsorbed position of a single Sc atom on B-PG, which was similar to the situation of the undoped-PG adsorbing Sc atom [42]. The optimized geometry is shown in Figure 2a. The Sc atom was located at the central hole of the Boron carbon six-membered ring and was slightly deviated. Simultaneously, the B-PG plane was slightly deformed, and the adsorption energy of the Sc atom on the B-PG was $-4.004 \mathrm{eV}$. In our previous study, the adsorption energy of a single Sc atom on clean PG was $-2.143 \mathrm{eV}$ [42], which was much smaller than that of single Sc on B-PG of $-4.004 \mathrm{eV}$. It can be seen that B doping can enhance the adsorption activity 
of the system; compared with pure porous graphene, the Sc atom was closer to the substrate and therefore had greater binding energy on the B-doped PG.

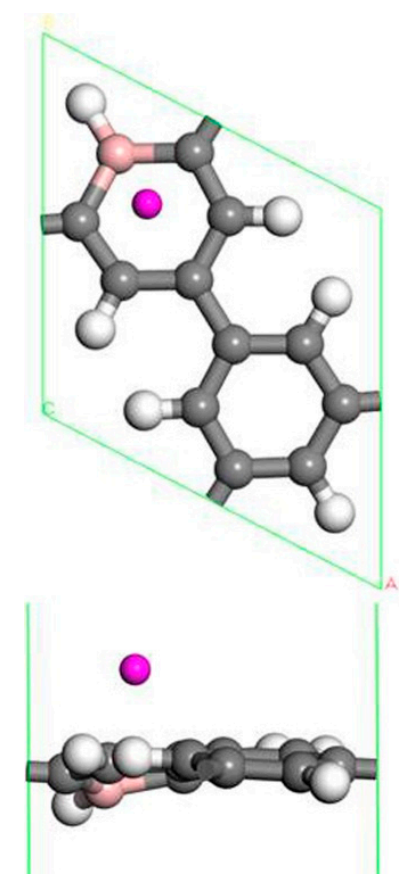

a
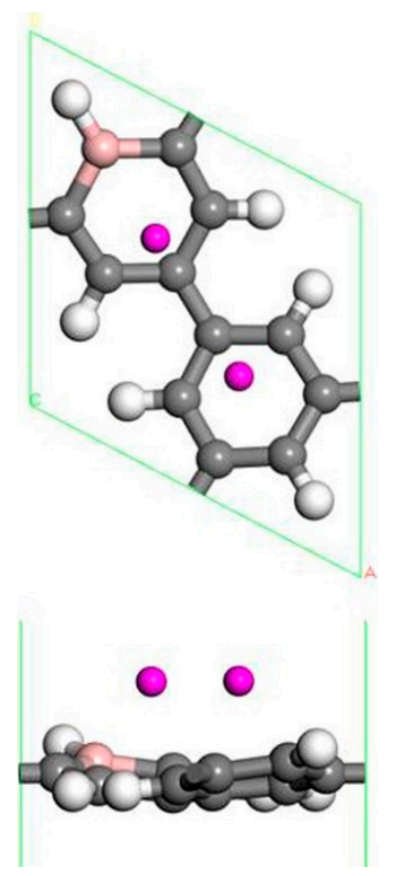

$\mathrm{b}$
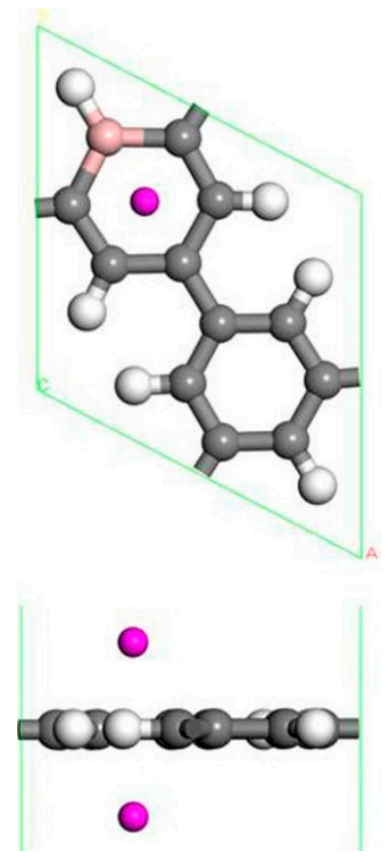

C

Figure 2. Sc-B/PG system optimized geometry: (a) Single Sc atom modification; (b) two Sc atoms single-sided modification; (c) two Sc atoms double-sided modified in the same pore position. Gray, white, pink, and purple spheres represent $\mathrm{C}, \mathrm{H}, \mathrm{B}$, and Sc atoms, respectively).

Figure 3 shows the partial densities of states (PDOS) of the Sc-B/PG system and the density of states of the clean PG system. It can be seen that the $2 \mathrm{p}$ orbit of the B atom and the $3 \mathrm{~d}$ orbit of the $\mathrm{Sc}$ atom overlaps in the interval of $-2.0 \sim-1.0 \mathrm{eV}$, indicating that there was a strong interaction between the $B$ atom and $S c$ atom. The $2 p$ orbit of the $C$ atom overlaps with the $3 \mathrm{~d}$ orbit of the Sc atom in the range of $-3.0 \sim-1.0 \mathrm{eV}$. In the interval of -1.0 to $0.0 \mathrm{eV}$, the peaks of the orbits of $\mathrm{C}$ and Sc atoms also overlap, meaning that the $\mathrm{C}$ and $\mathrm{Sc}$ atoms have an existing strong interaction. In addition, comparing the total density of states of the Sc-B/PG system with the clean PG system, we can see that the $3 \mathrm{~d}$ orbit of the Sc atom significantly changes the properties of the clean porous graphene near the Fermi surface; the peak of the Sc-B/PG system shifts to the left; and the electrons are close to the Fermi level, the system is more stable after doping the Sc and B atom. 


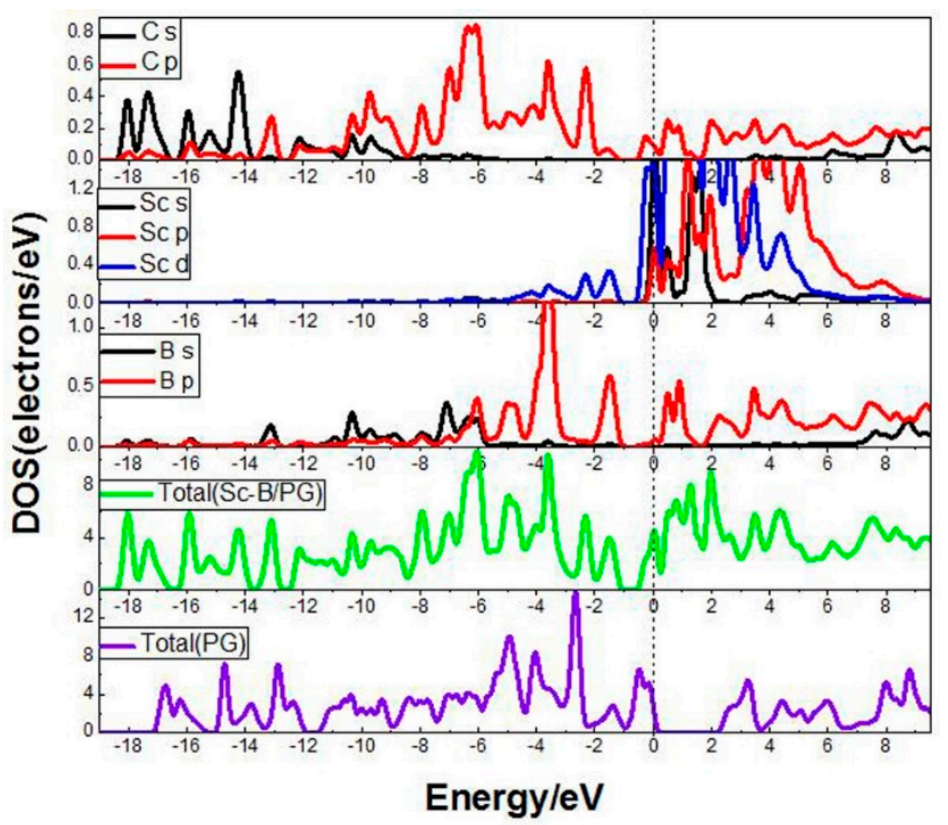

Figure 3. DOS of the Sc-B/PG system and PG system.

\subsubsection{The Adsorption of $\mathrm{H}_{2}$ on a Single Sc Atom Modified B-PG System}

A single Sc-modified B-doped porous graphene system can adsorb $5 \mathrm{H}_{2}$ molecules, and the optimized structures are given in Figure 4. Moreover, Table 2 lists the adsorption and average adsorption energy of $\mathrm{H}_{2}$, the distance between $\mathrm{Sc}$ atom and $\mathrm{H}_{2}$ molecules, and the distance between the $\mathrm{Sc}$ and $\mathrm{C}$ atom on the B-PG. A single $\mathrm{H}_{2}$ molecule has multiple adsorption sites on Sc-modified B-PG, including the $\mathrm{C}-\mathrm{C}$ bridge position, the $\mathrm{C}-\mathrm{H}$ bridge position, the $\mathrm{C}$ atom top position, and the $\mathrm{Sc}$ atom top position. It has been discovered that the most stable adsorption position of the first $\mathrm{H}_{2}$ molecule is the top position of the $\mathrm{C}$ atom. This $\mathrm{H}_{2}$ molecule has an adsorption energy of $-0.677 \mathrm{eV}$. Meanwhile, the $\mathrm{H}-\mathrm{H}$ bond was stretched to $0.868 \AA$. To further explore the hydrogen storage behavior of the system, $\mathrm{H}_{2}$ molecules were continuously added to the system. Table 2 indicates that $\mathrm{H}_{2}$ molecules' binding energies fluctuated with the number of adsorbed $\mathrm{H}_{2}$ molecules. The third $\mathrm{H}_{2}$ molecule adsorbed above the B-H bond and was slightly away from the hexagonal ring, so the adsorption energy decreased suddenly. However, the trend of $d_{S c-C}$ was a complete contrast to the trend of the $\mathrm{H}_{2}$ molecule adsorption energy. That is, the smaller the distance between Sc and the B-PG substrate, the greater the adsorption energy of hydrogen, and vice versa because the addition of hydrogen affects the position of Sc, and the position of Sc in turn affects the adsorption of hydrogen. The system eventually reaches a steady state through this mutually adjusted process. As a result of the symmetry of the $\mathrm{H}_{2}$ molecular bonding configuration, all $\mathrm{H}_{2}$ molecules were symmetrically distributed on every side of the $\mathrm{Sc}$ atom when the fourth $\mathrm{H}_{2}$ was adsorbed. The distance from the $\mathrm{H}_{2}$ molecule to the $\mathrm{Sc}$ atom always increased, but when the fourth $\mathrm{H}_{2}$ molecule adsorbed, the $d_{S c-H_{2}}$ suddenly decreased. At this time, the Sc atom was located at the center of the carbon ring doped by the B atom. Additionally, the first four $\mathrm{H}_{2}$ molecules were located on the same horizontal plane, which was parallel to the PG layer. Due to the limited space around the $\mathrm{Sc}$ atom and the repulsion between the adsorbed $\mathrm{H}_{2}$ molecules, the fifth $\mathrm{H}_{2}$ molecule moved to the upper layer after relaxation in Figure 4e. Therefore, the fifth $\mathrm{H}_{2}$ molecule had a $\mathrm{H}-\mathrm{H}$ bond length of $0.762 \AA$, and it had a minimum adsorption energy of $-0.187 \mathrm{eV}$, which was still bigger than $-0.093 \mathrm{eV}$ of the Sc-PG system without $\mathrm{B}$ doped [42]. The bond length of the adsorbed $\mathrm{H}_{2}$ molecule was in the range of $0.762 \sim 0.831 \AA$, and no dissociation of the $\mathrm{H}_{2}$ molecule was found. Therefore, the B doped PG decorated by Sc was more suitable for hydrogen storage at room temperature, and the practical application prospect was greater. The PG system modified by a single Sc atom can adsorb up to $5 \mathrm{H}_{2}$ molecules due to the doping of B atom. In a single Sc-modified B-doped 
porous graphene system, the average adsorption energy $\left(-0.515 \mathrm{eV} / \mathrm{H}_{2}\right)$ and the hydrogen storage capacity $(4.91 \mathrm{wt} . \%)$ are better than that of a Ti-PG system $(-0.486 \mathrm{eV})[43]$.

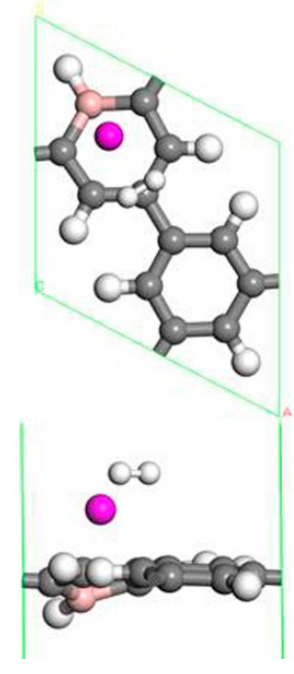

a

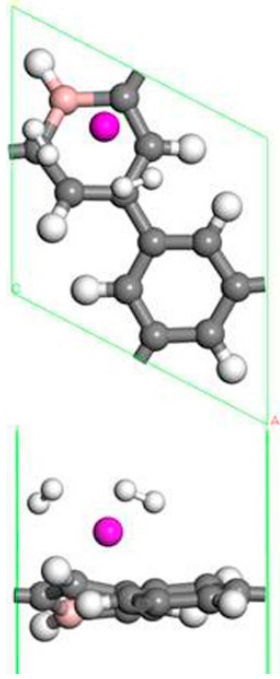

$\mathrm{b}$

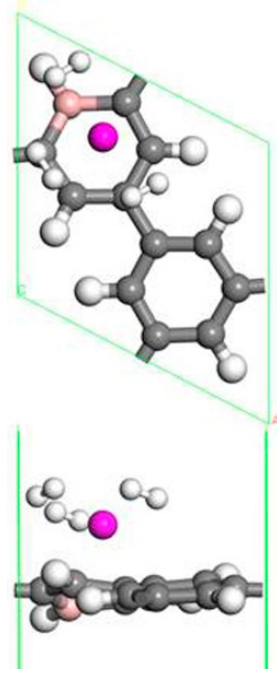

C

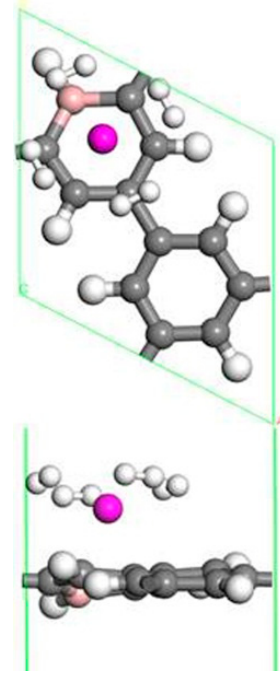

d

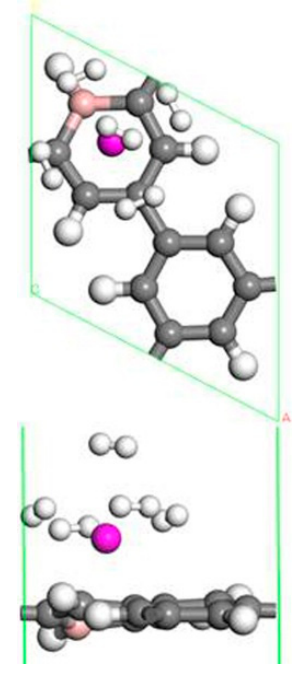

e

Figure 4. The optimized geometries of a single Sc-modified B-PG system with one $\mathrm{H}_{2}$ molecule (a), two $\mathrm{H}_{2}$ molecules (b), three $\mathrm{H}_{2}$ molecules (c), four $\mathrm{H}_{2}$ molecules (d) and five $\mathrm{H}_{2}$ molecules (e) adsorbed.

Table 2. Calculated adsorption energy and average adsorption energy of $\mathrm{H}_{2}$, distance between $\mathrm{Sc}$ atom and $\mathrm{H}_{2}$ molecules, and minimum distance between Sc atom and $\mathrm{C}$ atom in Sc-modified B-PG system.

\begin{tabular}{|c|c|c|c|c|c|}
\hline Number of $\mathbf{H}_{2}$ & $1 \mathrm{H}_{2}$ & $2 \mathrm{H}_{2}$ & $3 \mathrm{H}_{2}$ & $4 \mathrm{H}_{2}$ & $5 \mathrm{H}_{2}$ \\
\hline$E_{a d}(\mathrm{eV})$ & -0.677 & -0.682 & -0.416 & -0.614 & -0.187 \\
\hline$E_{a d}(\mathrm{eV})$ & -0.677 & -0.680 & -0.592 & -0.597 & -0.515 \\
\hline$d_{S c-H_{2}}(\AA)$ & 2.034 & 2.049 & 2.146 & 2.061 & 2.867 \\
\hline$d_{S c-C}(\AA)$ & 2.274 & 2.229 & 2.380 & 2.333 & 2.385 \\
\hline
\end{tabular}

Considering the interaction among the Sc atom with the adsorbed $\mathrm{H}_{2}$ molecule, we analyzed the partial densities of states (PDOS) of the $\mathrm{H}_{2}$ molecule and the Sc atom in Figure 5. It can be seen that the band broadening occurred in the $\mathrm{H}_{2}$ molecule from -11.0 to $-8.0 \mathrm{eV}$. In the interval of $-9.5 \sim-7.5 \mathrm{eV}$, the 1 s orbit of $\mathrm{H}_{2}$ overlapped with the $3 \mathrm{~d}$ orbit of $\mathrm{Sc}$, indicating that the 1 s orbit of $\mathrm{H}_{2}$ and the $3 \mathrm{~d}$ orbit of $\mathrm{Sc}$ had a existing interaction. When 1 4 $\mathrm{H}_{2}$ molecules were adsorbed on the Sc-decorated B-PG system, it could be seen that the peak of the $\mathrm{H}_{2}$ 1s orbit overlapped with the peak of the Sc $3 \mathrm{~d}$ orbit in the range of $-3.0 \sim 0.0 \mathrm{eV}$, which was in accordance with the strong adsorption energy of the $\mathrm{H}_{2}$ molecules (except the fifth hydrogen).

To further investigate the combined effects of the B atom and Sc atom on $\mathrm{H}_{2}$ molecule adsorption, the PDOS of one $\mathrm{H}_{2}$ molecule adsorbed in a single Sc-modified B-PG system was analyzed (as shown in Figure 6). From 1.0 to $0.0 \mathrm{eV}$, there were overlapping peaks between B 2p orbit, Sc $3 \mathrm{~d}$ orbit and $1 \mathrm{~s}$ orbit of the $\mathrm{H}_{2}$ molecule, which means that $\mathrm{B}$ and $\mathrm{Sc}$ both played a role in $\mathrm{H}_{2}$ adsorption. In addition, it can be observed that the $2 \mathrm{p}$ orbit of $\mathrm{B}$ atoms overlapped with the $3 \mathrm{~d}$ orbit of Sc atoms in most energy intervals, indicating that there was a strong interaction between B and Sc, which further explained why Sc had a higher binding energy in the B doping system. 


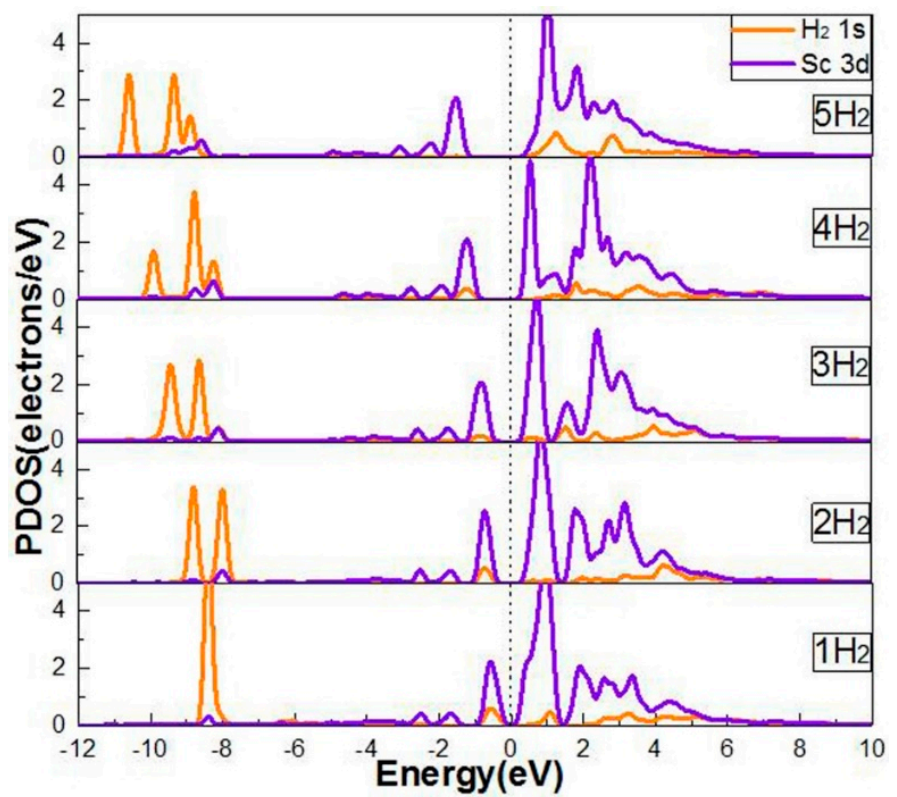

Figure 5. PDOS of Sc-decorated B-PG with one to five $\mathrm{H}_{2}$ molecules adsorbed.

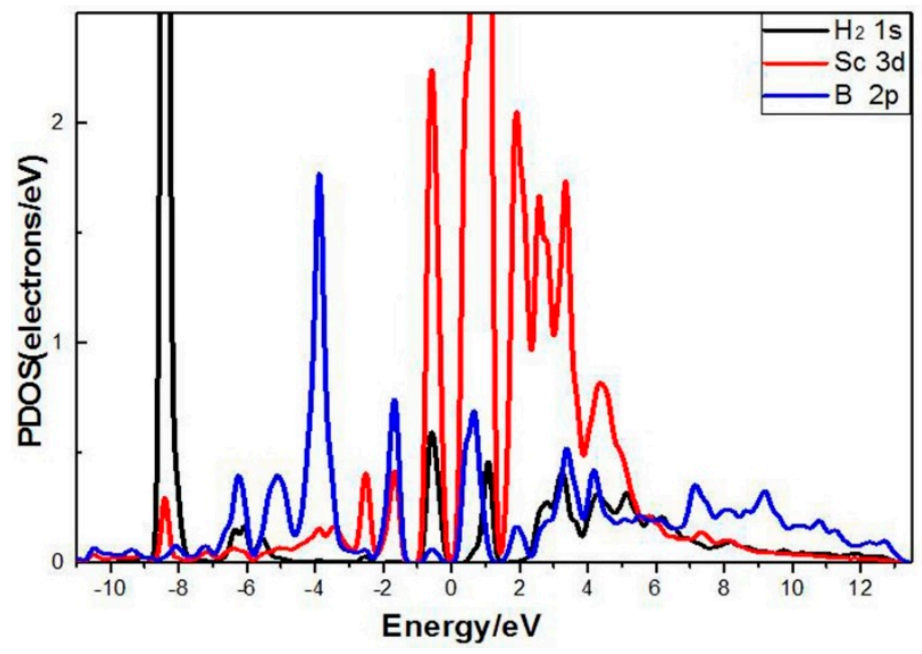

Figure 6. PDOS of Sc-decorated B-PG with one $\mathrm{H}_{2}$ molecules adsorbed.

The adsorption mechanism could be better understood by analyzing the charge density difference. Figure 7 shows the electronic charge density difference of the system after $\mathrm{H}_{2}$ molecules adsorbed. The blue and yellow isosurfaces represented the electron accumulation and electron loss regions, respectively, and the isosurface unit was $0.007 \mathrm{e} / A^{3}$. It can be observed from Figure $7 \mathrm{a}, \mathrm{b}$ that the yellow electron-depleting region was concentrated between the Sc atom and the B-PG, meaning that there was a large amount of charge transfer from the Sc atom to the B-PG. We also analyzed the Mulliken charge population before and after the adsorption of the Sc atom in the B-PG system. We found that the B atom $2 p$ orbit and the $C$ atom had an accumulation of electrons, while the $4 \mathrm{~s}$ orbit of $\mathrm{Sc}$ lost electrons by 1.5 e. Combined with the PDOS of Sc, B and C atom in Figure 3, it was found that the 4s orbit of Sc transferred some electrons to the $2 p$ orbit of $B$ and $C$, respectively. The charge population indicated that the $3 \mathrm{~d}$ orbit of Sc obtained 0.45 e electrons, which meant that the B-PG transferred electrons to the Sc atom. On the whole, the Sc carries a positive charge, and the B-PG layer carries a negative charge. Therefore, an electric field is formed between the Sc atom and the B-PG. The adsorption energy of Sc atoms on B-PG was large due to the strong electrostatic Coulomb interaction between Sc and B-PG, and the orbital interaction between Sc, B and C atoms. Since the Sc atom has a positive charge, 
this will attract the negative charge in $\mathrm{H}_{2}$ and accumulate near the metal. It can also be seen from Figure 7 that the blue electron region was concentrated between $\mathrm{H}_{2}$ and Sc. Near the side of the Sc atom, polarization occurred in the perpendicular direction of the $\mathrm{H}_{2}$ molecule. It has been indicated that the $\mathrm{H}_{2}$ molecule undergoes charge redistribution due to the electrostatic field between the Sc atom and the B-PG. Combined with the Mulliken charge population of the 1Sc-B-PG system it adsorbed two $\mathrm{H}_{2}$ molecules, the $\mathrm{H}$ atoms in the polarized $\mathrm{H}_{2}$ molecule were negatively charged at -0.17 e and -0.16 e. Conversely, the Sc atom was positively charged at $1.94 \mathrm{e}$. Therefore, there was a Coulomb attraction between the $\mathrm{H}_{2}$ molecule and the $\mathrm{Sc}$ atom. The $\mathrm{C}$ atom also had a partial charge transfer on B-PG, indicating that these $\mathrm{C}$ atoms also play a role on the $\mathrm{H}_{2}$ molecule adsorption. Consequently, the adsorption of $\mathrm{H}_{2}$ molecules in the Sc-modified B-PG system is mainly attributed to the orbital interaction between $\mathrm{H}$ and $\mathrm{Sc}$ atoms. Furthermore, the Coulomb attraction between $\mathrm{H}_{2}$ molecules (negatively charged) and Sc atoms (positively charged) strengthen the adsorption of $\mathrm{H}_{2}$ molecules.

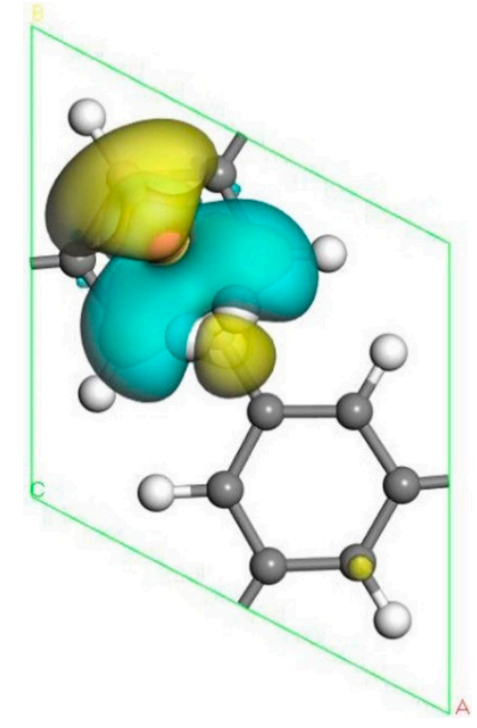

a

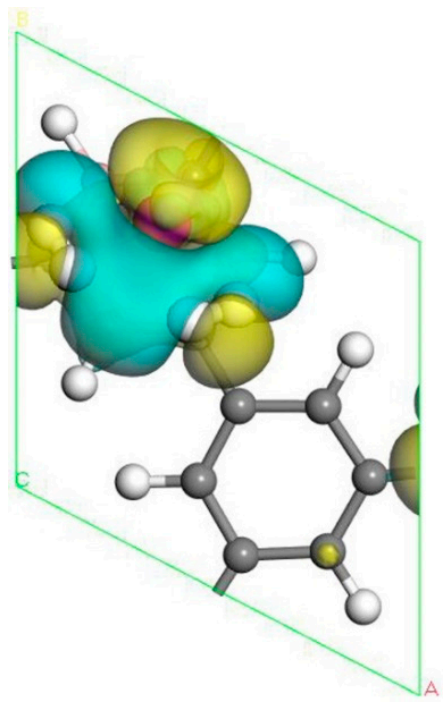

b

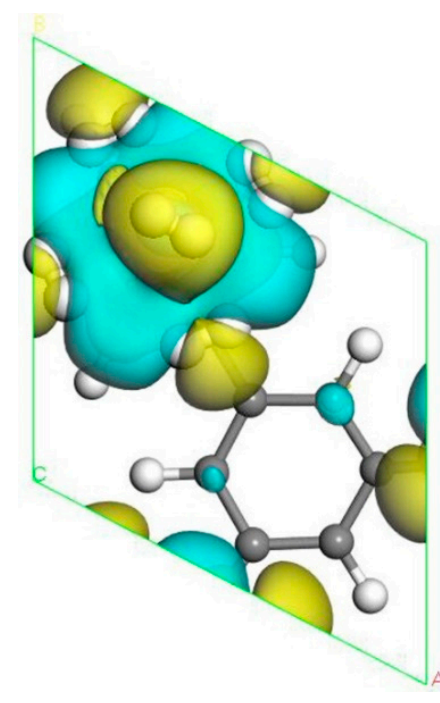

C

Figure 7. Electronic charge density difference for the Sc-B/PG system in the presence of (a) one $\mathrm{H}_{2}$ molecule; (b) two $\mathrm{H}_{2}$ molecules; (c) five $\mathrm{H}_{2}$ molecules.

To better understand the bonding environment of all B-H bonds in this structure after adsorption, we calculated a Bond Population of B-H bond and Mulliken charge Population of B, H, and Sc atoms in B-PG, Sc-B/PG and $\mathrm{H}_{2}$ adsorbed Sc-B/PG system, where $\mathrm{H}$ was the $\mathrm{H}$ atom in the first to fifth hydrogen molecules adsorbed. As shown in Table 3, the B-H population gradually increased as the hydrogen molecules continued to adsorb. After Sc atom modification, the positive charge of B atom decreased rapidly, indicating that $\mathrm{Sc}$ was stable on B-PG. Thereafter, the positive charge of the B atom fluctuated with the adsorption of hydrogen. The positive charge of $\mathrm{Sc}$ in the hydrogen storage process had been increasing. After the first $\mathrm{H}_{2}$ molecule was adsorbed by the Sc-B/PG structure, the Sc atom was charged to $1.72 \mathrm{e}$, which is greater than that of $1.56 \mathrm{e}$ in the undoped B atom system [42]. It was shown that after doping the B atom, the catalytic effect of Sc atoms in hydrogen storage was more obvious. As the hydrogen molecules continuously adsorbed, the $\mathrm{H}$ atom in $\mathrm{H}_{2}$ molecules started to be negatively charged and the charge amount gradually decreased. The $\mathrm{H}$ atom of the first $\mathrm{H}_{2}$ molecule after doping $\mathrm{B}$ atoms was charged $-0.17 \mathrm{e}$, and its absolute value was greater than that of $-0.13 \mathrm{e}$ in the undoped system [42]. This indicated that doping B atom enhanced the interaction between $\mathrm{H}_{2}, \mathrm{Sc}$ atoms and PG, which was more conducive to hydrogen storage. 
Table 3. Calculated Bond Population of the B-H bond and Mulliken Charge Population of B, H, Sc atoms in $\mathrm{B}-\mathrm{PG}, \mathrm{Sc}-\mathrm{B} / \mathrm{PG}$ and $\mathrm{H}_{2}$ adsorbed $\mathrm{Sc}-\mathrm{B} / \mathrm{PG}$ system.

\begin{tabular}{cccccccc}
\hline System & $\mathbf{B}-\mathbf{P G}$ & $\mathbf{S c - B / P G}$ & $\mathbf{1 H}_{\mathbf{2}}$ & $\mathbf{2 H}_{\mathbf{2}}$ & $\mathbf{3 H}_{\mathbf{2}}$ & $\mathbf{4} \mathbf{H}_{\mathbf{2}}$ & $\mathbf{5 H}_{\mathbf{2}}$ \\
\hline $\mathbf{B}-\mathbf{H}$ & 1.06 & 1.04 & 1.03 & 1.04 & 1.05 & 1.05 & 1.06 \\
$\mathbf{B}(\mathbf{e})$ & 0.33 & 0.04 & 0.04 & 0.03 & 0.06 & 0.06 & 0.05 \\
Sc (e) & None & 1.15 & 1.72 & 1.94 & 2.18 & 2.46 & 2.64 \\
$\mathbf{H ~ ( e ) ~}$ & None & None & -0.17 & -0.16 & -0.13 & -0.16 & -0.10 \\
\hline
\end{tabular}

\subsection{Two Sc Atoms Modified B-PG System}

\subsubsection{Adsorption Structure of two Sc Atoms Modified B-PG}

Transition metal atoms are prone to aggregation on the surface of graphene, resulting in a decrease in the free surface area of the graphene. Metal aggregation is mainly attributed to the high cohesive energy of the atoms [44-46]. The cohesive energy of the Sc atom is $3.900 \mathrm{eV}$ [47], each Sc atom is equivalent to an $\mathrm{H}_{2}$ adsorption site. To further increase the amount of hydrogen storage, a second Sc atom is added to the same side of the system. Figure $2 b$ shows one of the most stable geometries for adsorbing two Sc atoms on B-PG. It can be seen that the second Sc atom tended to adsorb at the center of the other carbon ring instead of agglomeration with the first $\mathrm{Sc}$ atom, which was attributed to the Coulomb repulsion between the two Sc atoms (the two Sc atoms have a positive charge of $0.90 \mathrm{e}$, $0.97 \mathrm{e}$, respectively), and there was a strong interaction between the Sc atom and the B-PG. The average binding energy of the two Sc atoms on B-PG was $-4.069 \mathrm{eV}$, and its absolute value was greater than the cohesive energy of the Sc atom, which was $3.900 \mathrm{eV}$. This means that the adsorption structure of the two Sc atoms on PG is more stable than that of the single Sc atom on B-PG.

The structure of two Sc atoms on both sides of B-PG was also studied to open up enough space to adsorb $\mathrm{H}_{2}$ molecules. Each transition metal atom is an active adsorption site. The double-sided modification of B-PG by the transition metal atom can raise the hydrogen storage area. On account of Figure 2a, the second Sc atom had four adsorption sites on the opposite side of the B-PG, including two carbon ring centers, $\mathrm{C}-\mathrm{C}$ bridge site, and half $\mathrm{C}$ ring pore sites on the opposite side. The most stable adsorption structure was obtained as shown in Figure 2c. The average binding energy of two Sc atoms was $-4.085 \mathrm{eV}$, larger than that of two Sc atoms in the clean PG system. Therefore, the presence of $B$ atoms in PG prevented the Sc atoms from aggregating. In addition, it can be seen from Figure $2 a, b$ that the B-PG system underwent slight deformation after Sc atoms were adsorbed. However, Figure 2c did not undergo any deformation, which was related to the symmetric distribution of Sc atoms.

\subsubsection{Hydrogen Storage of two Sc Atoms Modified B-PG}

The B-PG system with two Sc atoms modified on one side can adsorb $10 \mathrm{H}_{2}$ molecules. The optimized geometry structure is shown in Figure 8. The average adsorption energy of the B-PG system was about $-0.339 \mathrm{eV} / \mathrm{H}_{2}$ with the hydrogen storage capacity $(7.73 \mathrm{wt} . \%)$. Because the adsorption energy of two Sc atoms on B-PG was larger than their cohesion energy, the distance between them was close without bonding. 


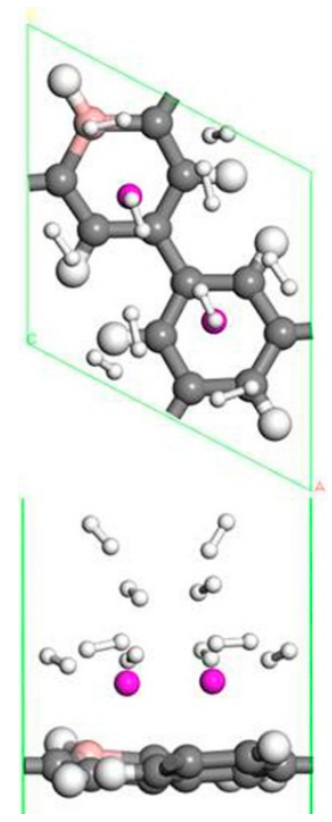

Figure 8. The optimized geometries for ten $\mathrm{H}_{2}$ molecules adsorbed on two Sc atoms single-sided modified B-PG.

Figure 2c is a stable structure of two Sc atoms modified B-PG on both sides, and the structure after hydrogen storage is shown in Figure 9. The distance between the two Sc atoms was large enough to prevent the aggregation where double-sided modified B-PG was concerned. There was no deformation that occurred in the B doped porous graphene surface, which was different from the distortion in the single Sc modified B-PG after hydrogen storage. The first eight $\mathrm{H}_{2}$ molecules were symmetrically distributed, but the ninth to twelfth $\mathrm{H}_{2}$ molecules gradually moved away from the $\mathrm{Sc}$ atoms and tended to adsorb to the top of the $\mathrm{C}-\mathrm{H}$ bond of adjacent carbon rings. Therefore, the average adsorption energy of $\mathrm{H}_{2}$ decreased. Two Sc double-sided modified B-PG systems can adsorb up to $12 \mathrm{H}_{2}$ molecules with an average adsorption energy of $-0.225 \mathrm{eV} / \mathrm{H}_{2}$. According to the standards of the U.S. Department of Energy and the International Energy Agency, the hydrogen storage capacity of ideal hydrogen storage materials should be greater than $5.50 \mathrm{wt} . \%$, and the adsorption energy between $\mathrm{H}_{2}$ molecules and materials should be from 0.200 to $0.700 \mathrm{eV}[48,49]$. Therefore, the average adsorption energy of the $\mathrm{H}_{2}$ molecule was also in the range of reversible hydrogen storage. The corresponding theoretical hydrogen storage capacity is $9.13 \mathrm{wt} . \%$, which is higher than the storage hydrogen content of the Sc-PG system 9.09 wt.\% [42] and is greater than the amount of hydrogen storage of the Y-PG system $7.87 \mathrm{wt} . \%$ [36]. The interaction between the $\mathrm{H}_{2}$ molecule, the Sc atom and the B-PG system in the two Sc atoms modified the B-PG system and was similar to the single Sc atom modification B-PG system, and is not described here. By comparing Figures $4 \mathrm{e}, 8$ and $9 \mathrm{f}$, it can be seen that the single Sc atom modified B-PG and the two Sc atoms single-sided modified B-PG adsorbed $\mathrm{H}_{2}$ molecules were layered. However, there was no hydrogen stratification with two Sc double-sided decorated B-PG. Moreover, the latter two $\mathrm{H}_{2}$ molecules adsorbed above the $\mathrm{C}-\mathrm{H}$ bond of another carbon six-membered ring. Therefore, the hydrogen storage spaces were larger in the Sc double-sided modification B-PG system than the $\mathrm{Sc}$ single-sided modification. In addition, the $\mathrm{H}_{2}$ molecules around a single $\mathrm{Sc}$ were parallel to the B-PG plane. When the two Sc atoms were modified on the same side, the $\mathrm{H}_{2}$ molecule changed from parallel to vertical, but they were not completely perpendicular to the B-PG surface due to the interaction. The perpendicular adsorption of $\mathrm{H}_{2}$ molecules is more likely to occur when two Sc atoms are modified at double sides, which is due to the interaction of $\mathrm{H}_{2}$ molecules on both sides of the B-PG. In summary, the two Sc located in the same boron-carbon ring position on the opposite side of B-PG is the most suitable structure for hydrogen storage, and Sc modified B-PG is a promising hydrogen storage material. 


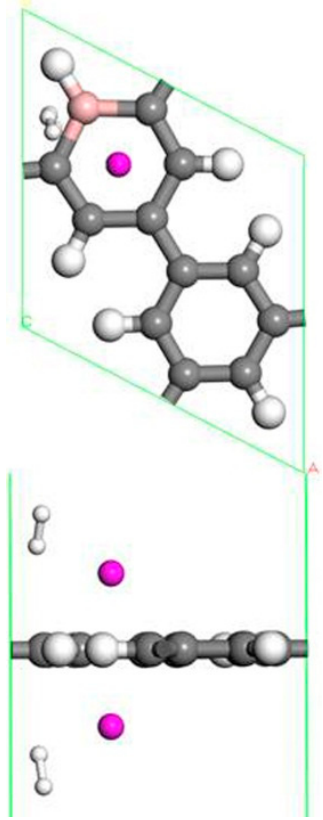

(a)

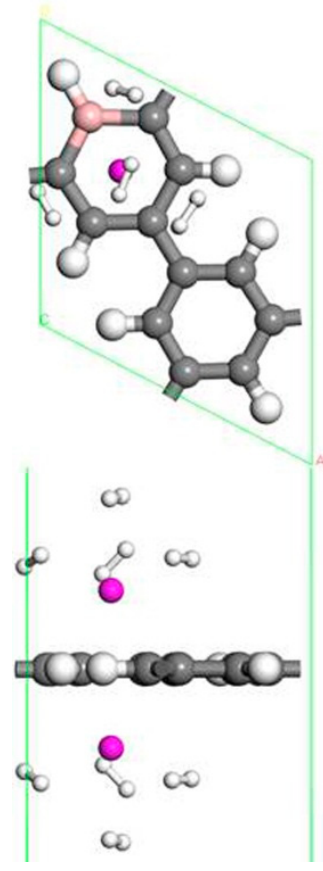

(d)

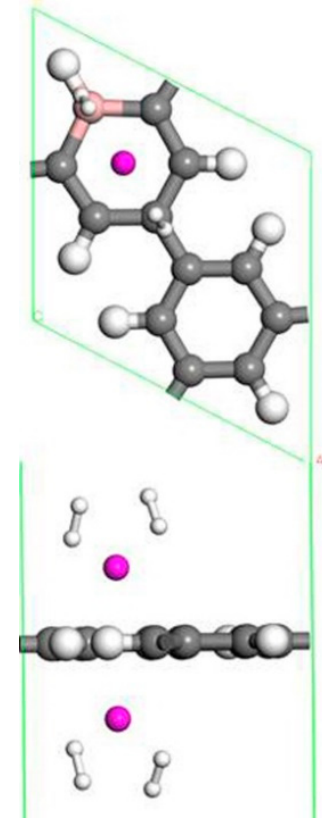

(b)

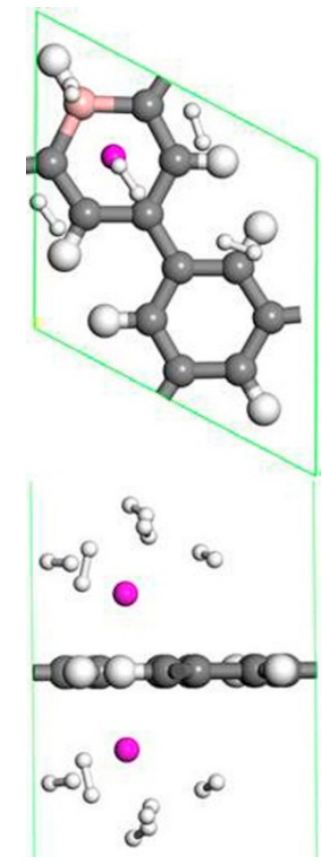

(e)

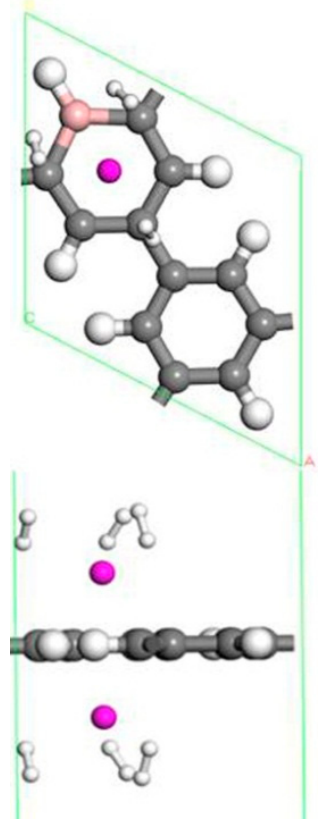

(c)

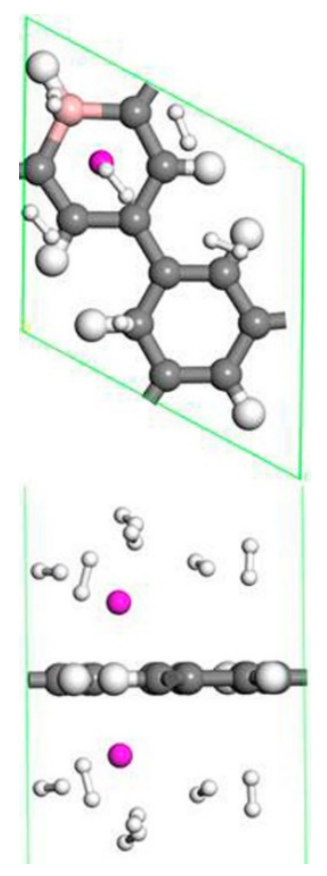

(f)

Figure 9. Optimized geometries of two Sc double-sided modified B-PG systems with two H2 molecules (a), four $\mathrm{H} 2$ molecules (b), six H2 molecules (c), eight $\mathrm{H} 2$ molecules (d), ten $\mathrm{H} 2$ molecules (e), and twelve $\mathrm{H} 2$ molecules (f) adsorbed.

\section{Conclusions}

We unambiguously investigated the hydrogen storage of the B atom doped Sc decorated PG. With an average adsorption energy $\left(-0.515 \mathrm{eV} / \mathrm{H}_{2}\right)$, the most stable adsorption position could adsorb five $\mathrm{H}_{2}$ molecules on the center of the boron-carbon ring for a single Sc atom system. By adding a second Sc atom to another side of the PG system, the hydrogen storage capacity effectively could be improved. 
When the two Sc atoms were located at both sides of the central boron-carbon hexagon, the theoretical hydrogen storage capacity could reach the maximum ( 9.13 wt.\%), which could adsorb twelve $\mathrm{H}_{2}$ molecules. At the same time, the two Sc atoms structure was the most suitable hydrogen storage for the Sc-B/PG system, possessing the average adsorption energy $\left(-0.225 \mathrm{eV} / \mathrm{H}_{2}\right)$. Furthermore, the adsorption of these $\mathrm{H}_{2}$ molecules in the Sc-modified B/PG system was also responsible for two aspects via the analysis of DOS and charge population: (i) The orbital interaction between $\mathrm{H}$ and Sc atoms; (ii) the Coulomb attraction between $\mathrm{H}_{2}$ molecules (negatively charge) and Sc atoms (positively charge). The $\mathrm{H}_{2}$ molecules were negatively charged due to polarization of the electrostatic field between $\mathrm{Sc}$ and B-PG. Therefore, Sc-modified B-doped porous graphene is expected to be used in the field of hydrogen storage.

Author Contributions: We would like to give great thanks to Y.C. because he designed the project. J.W. performed the calculations and prepared the manuscript, C.Z. revised the paper, L.Y. and M.Z. analyzed the data, and all authors discussed the results and commented on the manuscript.

Funding: This work was supported by the National Natural Science Foundation of China (No. 51562022) and the State Key Laboratory of Advanced Processing and Recycling of Non-ferrous Metals, Lanzhou University of Technology (No. SKLAB02014004).

Acknowledgments: The authors gratefully acknowledge the HongLiu first-class disciplines Development Program of the Lanzhou University of Technology.

Conflicts of Interest: The authors declare no conflicts of interest.

\section{References}

1. Lei, Y.; Shevlin, S.A.; Zhu, W.; Guo, Z.X. Hydrogen-induced magnetization and tunable hydrogen storage in graphitic structures. Phys. Rev. B 2008, 77. [CrossRef]

2. Du, A.; Zhu, Z.; Smith, S.C. Multifunctional Porous Graphene for Nanoelectronics and Hydrogen Storage: New Properties Revealed by First Principle Calculations. J. Am. Chem. Soc. 2010, 132, 2876-2877. [CrossRef] [PubMed]

3. Wang, Y.; Meng, Z.; Liu, Y.; You, D.; Wu, K.; Lv, J.; Wang, X.; Deng, K.; Rao, D.; Lu, R. Lithium decoration of three dimensional boron-doped graphene frameworks for high-capacity hydrogen storage. Appl. Phys. Lett. 2015, 106, 63901. [CrossRef]

4. Hussain, T.; Pathak, B.; Ramzan, M.; Maark, T.A.; Ahuja, R. Calcium doped graphane as a hydrogen storage material. Appl. Phys. Lett. 2012, 100, 183902. [CrossRef]

5. Song, N.; Wang, Y.; Zheng, Y.; Zhang, J.; Xu, B.; Sun, Q.; Jia, Y. New template for Li and Ca decoration and hydrogen adsorption on graphene-like SiC: A first-principles study. Comput. Mater. Sci. 2015, 99, 150-155. [CrossRef]

6. Gao, Y.; Zhao, N.; Li, J.; Liu, E.; He, C.; Shi, C. Hydrogen spillover storage on Ca-decorated graphene. Int. J. Hydrog. Energy 2012, 37, 11835-11841. [CrossRef]

7. Seenithurai, S.; Kodi Pandyan, R.; Vinodh Kumar, S.; Mahendran, M. H2 adsorption in Ni and passivated Ni doped $4 \AA$ single walled carbon nanotube. Int. J. Hydrog. Energy 2013, 38, 7376-7381. [CrossRef]

8. Bhattacharya, A.; Bhattacharya, S.; Majumder, C.; Das, G.P. Transition-Metal Decoration Enhanced Room-Temperature Hydrogen Storage in a Defect-Modulated Graphene Sheet. J. Phys. Chem. C 2010, 114, 10297-10301. [CrossRef]

9. Ramos-Castillo, C.M.; Reveles, J.U.; Cifuentes-Quintal, M.E.; Zope, R.R.; de Coss, R. Ti ${ }_{4}$ - and Ni $\mathrm{N}_{4}$-Doped Defective Graphene Nanoplatelets as Efficient Materials for Hydrogen Storage. J. Phys. Chem. C 2016, 120, 5001-5009. [CrossRef]

10. Ramos-Castillo, C.M.; Reveles, J.U.; Zope, R.R.; de Coss, R. Palladium Clusters Supported on Graphene Monovacancies for Hydrogen Storage. J. Phys. Chem. C 2015, 119, 8402-8409. [CrossRef]

11. Lebon, A.; Carrete, J.; Gallego, L.J.; Vega, A. Ti-decorated zigzag graphene nanoribbons for hydrogen storage. A van der Waals-corrected density-functional study. Int. J. Hydrog. Energy 2015, 40, 4960-4968. [CrossRef]

12. Chu, S.; Hu, L.; Hu, X.; Yang, M.; Deng, J. Titanium-embedded graphene as high-capacity hydrogen-storage media. Int. J. Hydrog. Energy 2011, 36, 12324-12328. [CrossRef] 
13. Liu, Y.; Ren, L.; He, Y.; Cheng, H.-P. Titanium-decorated graphene for high-capacity hydrogen storage studied by density functional simulations. J. Phys. Condens. Matter 2010, 22, 445301. [CrossRef]

14. Chi, S.-S.; Liu, Y.; Zhao, N.; Guo, X.; Nan, C.-W.; Fan, L.-Z. Solid polymer electrolyte soft interface layer with 3D lithium anode for all-solid-state lithium batteries. Energy Storage Mater. 2019, 17, 309-316. [CrossRef]

15. Luo, Z.; Fan, X.; Pan, R.; An, Y. A first-principles study of Sc-decorated graphene with pyridinic-N defects for hydrogen storage. Int. J. Hydrog. Energy 2017, 42, 3106-3113. [CrossRef]

16. Zhang, H.; Xia, G.; Zhang, J.; Sun, D.; Guo, Z.; Yu, X. Graphene-Tailored Thermodynamics and Kinetics to Fabricate Metal Borohydride Nanoparticles with High Purity and Enhanced Reversibility. Adv. Energy Mater. 2018, 8, 1702975. [CrossRef]

17. Chong, L.; Zeng, X.; Ding, W.; Liu, D.-J.; Zou, J. NaBH 4 in “Graphene Wrapper:" Significantly Enhanced Hydrogen Storage Capacity and Regenerability through Nanoencapsulation. Adv. Mater. 2015, 27, 5070-5074. [CrossRef] [PubMed]

18. Xia, G.; Tan, Y.; Chen, X.; Sun, D.; Guo, Z.; Liu, H.; Ouyang, L.; Zhu, M.; Yu, X. Monodisperse Magnesium Hydride Nanoparticles Uniformly Self-Assembled on Graphene. Adv. Mater. 2015, 27, 5981-5988. [CrossRef] [PubMed]

19. Yang, H.; Lombardo, L.; Luo, W.; Kim, W.; Züttel, A. Hydrogen storage properties of various carbon supported NaBH4 prepared via metathesis. Int. J. Hydrog. Energy 2018, 43, 7108-7116. [CrossRef]

20. Venkataramanan, N.S.; Suvitha, A.; Mizuseki, H.; Kawazoe, Y. A theoretical study of the effects of transition metal dopants on the adsorption and dissociation of hydrogen on nickel clusters. Int. J. Quantum Chem. 2013, 113, 1940-1948. [CrossRef]

21. Rojas, M.I.; Leiva, E.P.M. Density functional theory study of a graphene sheet modified with titanium in contact with different adsorbates. Phys. Rev. B 2007, 76. [CrossRef]

22. Faye, O.; Szpunar, J.A.; Szpunar, B.; Beye, A.C. Hydrogen adsorption and storage on Palladium-Functionalized graphene with NH-dopant: A first principles calculation. Appl. Surf. Sci. 2017, 392, 362-374. [CrossRef]

23. Durgun, E.; Ciraci, S.; Yildirim, T. Functionalization of carbon-based nanostructures with light transition-metal atoms for hydrogen storage. Phys. Rev. B 2008, 77. [CrossRef]

24. Kim, G.; Jhi, S.-H.; Park, N.; Louie, S.G.; Cohen, M.L. Optimization of metal dispersion in doped graphitic materials for hydrogen storage. Phys. Rev. B 2008, 78. [CrossRef]

25. Huang, H.-W.; Hsieh, H.-J.; Lin, I.-H.; Tong, Y.-J.; Chen, H.-T. Hydrogen Adsorption and Storage in Heteroatoms (B, N) Modified Carbon-Based Materials Decorated with Alkali Metals: A Computational Study. J. Phys. Chem. C 2015, 119, 7662-7669. [CrossRef]

26. Nachimuthu, S.; Lai, P.-J.; Jiang, J.-C. Efficient hydrogen storage in boron doped graphene decorated by transition metals-A first-principles study. Carbon 2014, 73, 132-140. [CrossRef]

27. Liu, W.; Liu, Y.; Wang, R. Prediction of hydrogen storage on Y-decorated graphene: A density functional theory study. Appl. Surf. Sci. 2014, 296, 204-208. [CrossRef]

28. Tokarev, A.; Avdeenkov, A.V.; Langmi, H.; Bessarabov, D.G. Modeling hydrogen storage in boron-substituted graphene decorated with potassium metal atoms: Hydrogen storage in boron-substituted graphene with potassium atoms. Int. J. Energy Res. 2015, 39, 524-528. [CrossRef]

29. Beheshti, E.; Nojeh, A.; Servati, P. A first-principles study of calcium-decorated, boron-doped graphene for high capacity hydrogen storage. Carbon 2011, 49, 1561-1567. [CrossRef]

30. Wang, H.; Zhou, Y.; Wu, D.; Liao, L.; Zhao, S.; Peng, H.; Liu, Z. Synthesis of Boron-Doped Graphene Monolayers Using the Sole Solid Feedstock by Chemical Vapor Deposition. Small 2013, 9, 1316-1320. [CrossRef]

31. Rangel, E.; Ramírez-Arellano, J.M.; Carrillo, I.; Magana, L.F. Hydrogen adsorption around lithium atoms anchored on graphene vacancies. Int. J. Hydrog. Energy 2011, 36, 13657-13662. [CrossRef]

32. Choudhary, A.; Malakkal, L.; Siripurapu, R.K.; Szpunar, B.; Szpunar, J. First principles calculations of hydrogen storage on $\mathrm{Cu}$ and Pd-decorated graphene. Int. J. Hydrog. Energy 2016, 41, 17652-17656. [CrossRef]

33. Bieri, M.; Treier, M.; Cai, J.; Aït-Mansour, K.; Ruffieux, P.; Gröning, O.; Gröning, P.; Kastler, M.; Rieger, R.; Feng, X.; et al. Porous graphenes: two-dimensional polymer synthesis with atomic precision. Chem. Commun. 2009, 6919-6921. [CrossRef] [PubMed]

34. Huang, C.; Wu, H.; Deng, K.; Tang, W.; Kan, E. Improved permeability and selectivity in porous graphene for hydrogen purification. Phys Chem Chem Phys 2014, 16, 25755-25759. [CrossRef] 
35. Reunchan, P.; Jhi, S.-H. Metal-dispersed porous graphene for hydrogen storage. Appl. Phys. Lett. 2011, 98, 93103. [CrossRef]

36. Yuan, L.; Chen, Y.; Kang, L.; Zhang, C.; Wang, D.; Wang, C.; Zhang, M.; Wu, X. First-principles investigation of hydrogen storage capacity of Y-decorated porous graphene. Appl. Surf. Sci. 2017, 399, 463-468. [CrossRef]

37. Lu, R.; Rao, D.; Meng, Z.; Zhang, X.; Xu, G.; Liu, Y.; Kan, E.; Xiao, C.; Deng, K. Boron-substituted graphyne as a versatile material with high storage capacities of Li and H2: a multiscale theoretical study. Phys. Chem. Chem. Phys. 2013, 15, 16120. [CrossRef]

38. Lu, R.; Rao, D.; Lu, Z.; Qian, J.; Li, F.; Wu, H.; Wang, Y.; Xiao, C.; Deng, K.; Kan, E.; et al. Prominently Improved Hydrogen Purification and Dispersive Metal Binding for Hydrogen Storage by Substitutional Doping in Porous Graphene. J. Phys. Chem. C 2012, 116, 21291-21296. [CrossRef]

39. Sun, J.; Wang, H.-T.; He, J.; Tian, Y. Ab initio investigations of optical properties of the high-pressure phases of ZnO. Phys. Rev. B 2005, 71. [CrossRef]

40. Rao, D.; Lu, R.; Meng, Z.; Wang, Y.; Lu, Z.; Liu, Y.; Chen, X.; Kan, E.; Xiao, C.; Deng, K.; et al. Electronic properties and hydrogen storage application of designed porous nanotubes from a polyphenylene network. Int. J. Hydrog. Energy 2014, 39, 18966-18975. [CrossRef]

41. Rao, D. Improve hydrogen storage capacities of several porous materials. Ph.D. Thesis, Nanjing University of Science Technology, Nanjing, China, 2013; p. 68.

42. Chen, Y.; Wang, J.; Yuan, L.; Zhang, M.; Zhang, C. Sc-Decorated Porous Graphene for High-Capacity Hydrogen Storage: First-Principles Calculations. Materials 2017, 10, 894. [CrossRef] [PubMed]

43. Yuan, L.; Kang, L.; Chen, Y.; Wang, D.; Gong, J.; Wang, C.; Zhang, M.; Wu, X. Hydrogen storage capacity on Ti-decorated porous graphene: First-principles investigation. Appl. Surf. Sci. 2018, 434, 843-849. [CrossRef]

44. Knick, C.R. Modeling the Exfoliation Rate of Graphene Nanoplatelet Production and Application for Hydrogen Storage. Master's Thesis, Wright State University, Dayton, OH, USA, 2012; pp. 52-60.

45. Ao, Z.; Dou, S.; Xu, Z.; Jiang, Q.; Wang, G. Hydrogen storage in porous graphene with Al decoration. Int. J. Hydrog. Energy 2014, 39, 16244-16251. [CrossRef]

46. Nam, H.; Jang, S.; Lee, W. Strategies for the Modification of Adhesion at Graphene/Al Interfaces: A First Principles Study. J. Nanosci. Nanotechnol. 2014, 14, 7674-7678. [CrossRef] [PubMed]

47. Chen, M.; Yang, X.-B.; Cui, J.; Tang, J.-J.; Gan, L.-Y.; Zhu, M.; Zhao, Y.-J. Stability of transition metals on Mg (0001) surfaces and their effects on hydrogen adsorption. Int. J. Hydrog. Energy 2012, 37, 309-317. [CrossRef]

48. Zhang, Z.W.; Li, J.C.; Jiang, Q. Hydrogen Adsorption on Eu/SWCNT Systems: A DFT Study. J. Phys. Chem. C 2010, 114, 7733-7737. [CrossRef]

49. Sahaym, U.; Norton, M.G. Advances in the application of nanotechnology in enabling a "hydrogen economy". J. Mater. Sci. 2008, 43, 5395-5429. [CrossRef]

Sample Availability: Samples of the compounds are not available from the authors.

(C) 2019 by the authors. Licensee MDPI, Basel, Switzerland. This article is an open access article distributed under the terms and conditions of the Creative Commons Attribution (CC BY) license (http://creativecommons.org/licenses/by/4.0/). 